\title{
Direct plasmon-driven photoelectrocatalysis
}

Hossein Robatjazi, ${ }^{\dagger, \|, \perp}$ Shah Mohammad Bahauddin, ${ }^{\dagger, \|, \perp}$ Chloe Doiron, ${ }^{\dagger}, \|, \perp$ Isabell Thomann $*, \dagger, t, \delta, \|, \perp$

Department of Electrical and Computer Engineering, ${ }^{t}$ Department of Materials Science and NanoEngineering, ${ }^{\S}$ Department of Chemistry, "Laboratory for Nanophotonics, and $\perp$ Rice Quantum Institute, Rice University, 6100 Main Street, Houston, Texas 77005, United States.

\section{Corresponding Author}

* Email: isabell.thomann@,rice.edu 
Abstract

Harnessing the energy from hot charge carriers is an emerging research area with the potential to improve energy conversion technologies ${ }^{12}$. Here we present a novel plasmonic photoelectrode architecture carefully designed to drive photocatalytic reactions by efficient, non-radiative plasmon decay into hot carriers. In contrast to past work, our architecture does not utilize a Schottky junction - the commonly used building block to collect hot carriers. Instead, we observed large photocurrents from a Schottky-free junction due to direct hot electron injection from plasmonic gold nanoparticles into the reactant species upon plasmon decay. The key ingredients of our approach are (i) an architecture for increased light absorption inspired by optical impedance matching concepts ${ }^{4}$ (ii) carrier separation by a selective transport layer and (iii) efficient hot-carrier generation and injection from small plasmonic $\mathrm{Au}$ nanoparticles to adsorbed water molecules. We also investigated the quantum efficiency of hot electron injection for different particle diameters to elucidate potential quantum effects while keeping the plasmon resonance frequency unchanged. Interestingly, our studies did not reveal differences in the hotelectron generation and injection efficiencies for the investigated particle dimensions and plasmon resonances. 
KEYWORDS: Plasmonic solar energy conversion, hot-electron, quantum efficiency, solar water splitting, $\mathrm{Au} / \mathrm{NiO}_{\mathrm{x}}$, selective transport layer

Plasmonic metal nanostructures are currently receiving considerable attention in the quest for efficient solar-to-fuel energy conversion, in large part owing to their unique light harnessing capabilities associated with their localized surface plasmon resonance (LSPR) features 35678910 111213141516 . LSPR-assisted solar-to-fuel energy conversion ${ }^{31718}$ has been shown to utilize three primary mechanisms, (I) elastic light scattering by plasmonic particles, (II) plasmonic near-field energy transfer ${ }^{6}$, and (III) non-radiative plasmon decay into hot carriers 819202122232425 . In particular this last mechanism is emerging as a hot topic, since the utilization of hot carriers to drive chemical reactions may open up pathways that are inaccessible by conventional methods such as heating or applying an electrochemical bias ${ }^{2}{ }^{26}$. Past demonstrations of hot-carrier utilization focused on employing a Schottky barrier for hot-electron extraction. In this scenario, only hot-electrons with sufficient energy to overcome the Schottky barrier can be collected from the conduction band of the semiconductor 581427 and spatially separated from the holes left behind in the metal nanostructure. Such Schottky barriers have been the central building block for harnessing hot carriers in plasmon-based photodetectors ${ }^{28}{ }^{29}$, sensors ${ }^{30}$, photovoltaic and photocatalytic devices ${ }^{226313233}$.

In this paper, we describe an alternative architecture to achieve efficient and direct injection of hot electrons from plasmonic gold nanoparticles to adsorbed water molecules for solar water splitting in a Schottky junction-free device. Fig. 1a shows our plasmonic photoelectrode structure. Here, carrier separation is facilitated by a selective transport layer. The resulting enforced confinement of electrons within the nanoparticles, i.e., in direct contact with water, opens up a new pathway for direct injection of hot electrons into the unoccupied orbitals of 
adsorbed molecules. We also experimentally investigated the quantum efficiency of hot electron injection into water molecules employing anisotropic gold nanoparticles (Au NPs) to reveal potential quantum effects. Our studies were inspired by recent theoretical work that showed that the hot-electron generation efficiency should depend critically on the plasmonic particle size ${ }^{34}$. We investigated two samples consisting of Au hemispheres with distinct dimensions: (i) $30 \mathrm{~nm}$ diameter, $6 \mathrm{~nm}$ thick, and (ii) $10 \mathrm{~nm}$ diameter, $2 \mathrm{~nm}$ thick. We will henceforth refer to these as $30 \mathrm{~nm}$ and $10 \mathrm{~nm}$ diameter particles, respectively. Interestingly, our studies did not reveal differences in hot-electron generation and injection efficiencies between these two samples.

The utilization of hot electrons has generally been hampered by their extremely short relaxation lifetimes due to electron-phonon interactions ${ }^{34}$ and other fast relaxation processes. Hence, hot carriers need to be harvested on femtosecond to picosecond timescales. Recent work in the area of solar water splitting ${ }^{11}$ and photocatalytic $\mathrm{CO}_{2}$ reduction ${ }^{35}$ has begun to investigate the efficiency and the lifetime of hot-electrons generated via plasmon decay at semiconductor/metal Schottky interfaces. Kim et al. ${ }^{13}$ studied Au particles larger than $50 \mathrm{~nm}$ and observed that the quality factor of the plasmon resonance is directly related to the photocatalytic activity of the particles. Qian et. al. ${ }^{36}$ found that small plasmonic Au particles cannot inject a sufficient number of hot electrons into the conduction band of $\mathrm{TiO}_{2}$ to raise the energy level sufficiently to drive the hydrogen evolution reaction (HER). DuChene et. al. ${ }^{21}$ found an increased lifetime of excited-state carriers in plasmonic $\mathrm{Au} / \mathrm{TiO}_{2}$ heterostructures compared to bare $\mathrm{TiO}_{2}$. And recently, plasmon-induced hot carrier generation has culminated in a report demonstrating an autonomous device for solar water splitting based on a plasmonic gold nanorod array/ $/ \mathrm{TiO}_{2}$ Schottky junction ${ }^{8}$. Unfortunately, the injection efficiency of hot carriers at a Schottky interface is extremely low, in large part owing to the momentum mismatch of hot 
electrons traversing the junctions ${ }^{37}$. Therefore, novel architectures that avoid a Schottky interface can potentially improve the efficiency of hot electron devices. The efficiency could be further increased if momentum conservation can be relaxed, e.g. by hot electron injection from small-sized plasmonic particles into molecules. Smaller metal nanoparticles are also advantageous since they facilitate the efficient decay of plasmons into energetic hot carriers ${ }^{34}$. From an optical perspective small plasmonic particles, however, have a smaller absorption cross section than larger particles making it challenging to achieve complete light absorption, and correspondingly efficient hot carrier generation, within these particles. From an electrical standpoint charge recombination must be avoided by fast separation of oppositely charged carriers. Here, we describe a novel plasmonic photoelectrode architecture that achieves a combination of high optical absorption within small Au NPs, efficient charge separation by a hole-transporting but electron-blocking layer, and efficient hot carrier injection into adsorbed water molecules.

Figure $1 \mathrm{~b}$ shows the energy schematic of our structure. Here, sun light resonantly excites plasmons in gold nanoparticles, which subsequently decay into hot electrons. The hot electrons are directly injected into water molecules to drive the hydrogen evolution reaction. The electronic function of the $\mathrm{NiO}_{\mathrm{x}}$ layer ${ }^{38}$ is to block electron transport while allowing for hole transport to the aluminum back electrode and further through the external circuit to the platinum counter electrode. Our architecture is also designed to maximize light absorption within the layer of small gold nanoparticles. Here, our design is inspired by optical impedance matching concepts ${ }^{43}$, where the optical function of $\mathrm{NiO}_{\mathrm{x}}$ is to serve as a wide bandgap spacer layer between the plasmonic nanoparticles and the aluminum back reflector. The spacer layer thickness can be adjusted to achieve maximum absorption within the gold nanoparticles based on electromagnetic 
simulations. To absorb a broader range of the solar spectrum we employed a heterogeneous particle size distribution exhibiting a corresponding spread of plasmon resonance wavelengths.

As we will demonstrate in this article, our device is capable of efficient direct hot electron injection for photocatalysis, and the produced photocurrent is similar to that of recent reports ${ }^{8}$ for driving the hydrogen evolution reaction, but in contrast to previous designs, our device architecture possesses a relatively small catalytic surface area (basically a planar geometry), it does not utilize expensive platinum as a co-catalyst, it uses a minimum amount of gold, and importantly, it does not rely on a Schottky junction to collect the hot-electrons, thereby opening possibilities for cheaper, simpler and more efficient devices.

To investigate the mechanism of plasmon mediated photocatalysis in our Schottky junctionfree plasmonic photocathode, we took wavelength-resolved photocurrent measurements in a range from $375 \mathrm{~nm}$ to $950 \mathrm{~nm}$. These measurements were performed in a three-electrode photoelectrochemical cell in a nitrogen-purged solution of $0.5 \mathrm{M} \mathrm{Na}_{2} \mathrm{SO}_{4}$ buffered at $\mathrm{pH}$ 5.2. The photocathode consisted of $\mathrm{NiO}_{\mathrm{x}} / \mathrm{Al}$ samples decorated with polydisperse Au nanoparticle size distributions (with diameters of most frequent occurrence) centered around $10 \mathrm{~nm}$ and $30 \mathrm{~nm}$, respectively (see Supporting Information for detailed fabrication process). SEM images show the Au nanoparticles (Figure $2 \mathrm{~b}, \mathrm{c}$ ) formed from annealing different thicknesses $(2 \mathrm{~nm}, 6 \mathrm{~nm})$ of $\mathrm{Au}$ films on $\mathrm{NiO}_{\mathrm{x}}$ and the insets show the corresponding particle size distributions. We found that evaporation of a nominal thickness of $2 \mathrm{~nm} \mathrm{Au}$ on top of a $40 \mathrm{~nm}$ thick $\mathrm{NiO}_{\mathrm{x}}$ film and subsequent annealing results in the formation of well-separated particles with a diameter of 10 $\mathrm{nm}$ and a narrow particle size distribution (Fig. 2b). For $6 \mathrm{~nm}$ thick Au, we obtain particle diameters around $30 \mathrm{~nm}$ with a broader particle size distribution (Fig. 2c) however this distribution does not contain the smallest particle diameters (i.e. 2 to $5 \mathrm{~nm}$ diameter Au NPs) that 
are only present in the $2 \mathrm{~nm}$ thick evaporated Au films. When we increased the evaporation thickness to $10 \mathrm{~nm}$, large gold nanoislands with typical sizes larger than $150 \mathrm{~nm}$ were formed after annealing of the sample (Supporting Information S2).

A proper insight into the possibility of direct hot-electron generation and injection into water after non-radiative plasmon decay can be gained by comparing the wavelength-resolved incident-photon-to-current conversion efficiency (IPCE) with numerically simulated absorption spectra. This comparison is provided in Fig. 2a. For both samples $(10 \mathrm{~nm}$ and $30 \mathrm{~nm}$ diameter $\mathrm{Au}$ NPs) the measured IPCE maxima match with the simulated absorbed fraction within the gold NPs. The measured IPCE for $30 \mathrm{~nm}$ Au NPs is approximately three times as large as that of the $10 \mathrm{~nm}$ Au NPs. We performed electromagnetic simulations of light absorption within the gold nanoparticles using the finite difference time domain (FDTD) method. For our electromagnetic simulations we chose the most frequently occurring particle diameters in our samples $(30 \mathrm{~nm}$ and $10 \mathrm{~nm}$, respectively) based on the experimentally determined particle size distribution in our scanning electron microscope (SEM) images shown in Fig. 2b and 2c. Both numerical structures employ gold nanoparticles with an anisotropic shape with a 5:1 diameter-to-thickness aspect ratio (30 nm: $6 \mathrm{~nm}$ and $10 \mathrm{~nm}: 2 \mathrm{~nm}$, respectively). The anisotropic shape is the reason for the plasmon resonance being shifted to the near-infrared around $800 \mathrm{~nm}$. We initially performed finite difference time domain (FDTD) simulations to maximize light absorption within the $30 \mathrm{~nm}$ diameter, $6 \mathrm{~nm}$ thick gold nanoparticles by optimizing the $\mathrm{NiO}_{\mathrm{x}}$ spacer layer thickness.

The numerical absorbed fraction reaches about $80 \%$ for the $30 \mathrm{~nm}$ particles and about $50 \%$ for the $10 \mathrm{~nm}$ particles for the same $\mathrm{NiO}_{\mathrm{x}}$ spacer layer thickness of $40 \mathrm{~nm}$ (Supporting Information S9). The absorbed fraction in the $10 \mathrm{~nm}$ Au NPs could be similar high as for the $30 \mathrm{~nm}$ Au NPs by employing an $80 \mathrm{~nm}$ thick $\mathrm{NiO}_{\mathrm{x}}$ spacer layer (Supporting Information S9) but here we 
decided to keep the $\mathrm{NiO}_{\mathrm{x}}$ film thickness the same in order to keep the electronic properties for both samples the same. As expected, the simulated absorbed fraction within the Au NPs shows increased absorption for both samples at wavelengths below $525 \mathrm{~nm}$ due to interband transitions from the occupied d-band. However, the absorption due to interband transitions does not result in a measurable cathodic photocurrent in the $450 \mathrm{~nm}$ to $550 \mathrm{~nm}$ wavelength region. The reason is that electrons excited from the d-band are promoted to unoccupied states near the Fermi level, and therefore are not energetic enough to drive the hydrogen evolution reaction (see Figure 1b). We observe a small photocurrent below $425 \mathrm{~nm}$ close to the band gap of $\mathrm{NiO}_{\mathrm{x}}$, which we attribute to light absorption in the $\mathrm{NiO}_{\mathrm{x}}$.

We performed control experiments to establish that the measured photocurrent arose from a direct electron transfer from Au NPs to protons in water, rather than from any enhancement in the $\mathrm{NiO}_{\mathrm{x}}$. We carried out cyclic voltammetry measurements in structures with and without $\mathrm{Au}$ nanoparticles. At an electrode potential of $0 \mathrm{~V}$ vs. RHE, we observed photocurrents of $25 \mu \mathrm{A} / \mathrm{cm}^{2}$ with Au NPs (Fig. 2d) and $0.250 \mu \mathrm{A} / \mathrm{cm}^{2}$ without Au NPs (Supporting Information S8, Fig. a), respectively. Thus, the photocurrents without Au NPs are $\sim 100 \mathrm{x}$ smaller than with Au NPs. We also conducted wavelength-resolved photocurrent measurements of our $\mathrm{NiO}_{\mathrm{x}} / \mathrm{Al}$ samples without $\mathrm{Au}$ NP in the wavelength range from $375 \mathrm{~nm}$ to $900 \mathrm{~nm}$ at an electrode potential of $-0.1 \mathrm{vs}$. the RHE. No photocurrent was observed from $500 \mathrm{~nm}$ to $900 \mathrm{~nm}$ demonstrating that the photocurrent observed in the Au-containing structure does not originate from defect or trap states in the $\mathrm{NiO}_{\mathrm{x}}$. However, a photocurrent of approximately $-0.25 \mu \mathrm{A} / \mathrm{cm}^{2}$ is found at $375 \mathrm{~nm}$, which is near the bandgap of $\mathrm{NiO}_{\mathrm{x}}$ (Supporting Information S8 Fig. b). We also performed Mott Schottky measurements of our $40 \mathrm{~nm}$ thick $\mathrm{NiO}_{\mathrm{x}}$ film (Supporting Information S6), and found that the material is intrinsically p-type with a hole concentration of $10^{18}-10^{19} \mathrm{~cm}^{-3}$ and its flat 
band potential lies very close to the Fermi level of gold. This means, the Fermi level of the gold nanoparticles is very close to the valence band of $\mathrm{NiO}_{\mathrm{x}}$ and thus no charge transfer to a trap state near the conduction band (which is almost $3.5 \mathrm{eV}$ away) can be driven by a plasmon resonance of $800 \mathrm{~nm}(\approx 1.55 \mathrm{eV})$.

Given that these control experiments did not produce appreciable photocurrents, we propose the following mechanism for the observed IPCE of $0.1 \%$ : Light absorption is enhanced by exciting localized surface plasmon resonances in a gold nanoparticle/spacer/reflector geometry. Plasmon quanta decay into electron-hole pairs. The plasmon resonance frequency of our photocathode lies at around $800 \mathrm{~nm}-825 \mathrm{~nm}$, thus we expect a maximum energy of $\sim 1.55 \mathrm{eV}$ for hot-electrons generated from the surface plasmon resonance. The electrons possess a nonequilibrium Fermi-Dirac distribution where the tail of the distribution has enough energy to overcome the energy barrier required for driving the hydrogen evolution reaction with a redox potential that lies at excitation energy of about $1 \mathrm{eV}$ above the Fermi energy of the Au NP. In other words, hot electrons with energies greater than $1 \mathrm{eV}$ plus the required overpotential should be able to transfer directly into the unoccupied states of the reactant species and thus result in the initiation of the photocatalytic HER. The favorable band edges of $\mathrm{p}-\mathrm{NiO}_{\mathrm{x}}$ allow for hole transfer while blocking electron injection. The holes collected by $\mathrm{NiO}_{\mathrm{x}}$ are then collected by underlying aluminum and transported to the counter electrode to balance the reaction. Although the nature of carrier transport in $\mathrm{NiO}_{\mathrm{x}}$ has not been fully unraveled, a growing consensus supports the small polaron hopping mechanism ${ }^{40}$.

Figure $2 \mathrm{~d}$ shows the current versus potential of a plasmonic photocathode decorated with 10 $\mathrm{nm}$ diameter nanoparticles under chopped illumination at one sun $\left(100 \mathrm{~mW} / \mathrm{cm}^{2}\right)$ immersed in a nitrogen-purged solution of $0.5 \quad \mathrm{M} \mathrm{Na} \mathrm{Na}_{2} \mathrm{SO}_{4}$ buffered at $\mathrm{pH}$ 5.2. A three-electrode 
photoelectrochemical cell was used with $\mathrm{Ag} / \mathrm{AgCl}$ (in sat. $3.0 \mathrm{M} \mathrm{KCl}$ ) and a $\mathrm{Pt}$ mesh as the reference and the counter electrode, respectively. The photocurrent is approximately $2.6 \mu \mathrm{A} / \mathrm{cm}^{2}$ at $0.5 \mathrm{~V}$ vs RHE and reaches $24 \mu \mathrm{A} / \mathrm{cm}^{2}$ at zero versus the RHE. We also performed chronoamperometry measurements at $-0.1 \mathrm{~V}$ vs. RHE and observed a photocurrent of $26 \mu \mathrm{A} / \mathrm{cm}^{2}$ for $30 \mathrm{~nm}$ diameter Au NPs and of $13 \mu \mathrm{A} / \mathrm{cm}^{2}$ for $10 \mathrm{~nm}$ diameter Au NPs at a light intensity of $100 \mathrm{~mW} \mathrm{~cm}^{-2}$ (Supporting Information S7). These observed photocurrents are on a par with a recently demonstrated large catalytic surface area $\mathrm{TiO}_{2}$-coated plasmonic gold nanorod array Schottky junction geometry that in addition added platinum nanoparticles to catalyze the HER reaction ${ }^{8}$.

The absorption spectrum of a plasmonic device is generally influenced by the size, shape, environment and distribution of the plasmonic particles and thus these are important parameters for tuning plasmon-mediated catalysis. These parameters do not only affect the wavelength of the plasmon resonance but also the efficiency of hot carrier generation and injection ${ }^{2,34,41}$. In Figure 3 we show a comparison of the internal quantum efficiency (IQE) for $10 \mathrm{~nm}$ diameter and $30 \mathrm{~nm}$ diameter Au particles. The solid curve shows the internal quantum efficiency (left axis), and the symbols (right axis) show the measured photocurrent. The IQE was calculated from the measured photocurrent and the measured absorbed fraction (Fig. 4). We estimated the error bars based on the measurement of our two best samples for $10 \mathrm{~nm}$ and $30 \mathrm{~nm}$ diameter $\mathrm{Au}$ nanoparticles respectively, where we considered the standard deviation and associated error propagation for each independent variable (i.e. photocurrent, incident light intensity, intensity of the reflected light). The IQE for $10 \mathrm{~nm}$ and $30 \mathrm{~nm}$ diameter particles is the same, demonstrating that both size particles are equally efficient in terms of hot electron generation and injection. The observation of an identical IQE for these two different particle sizes is surprising in light of 
recent results from Manjavacas et al ${ }^{34}$. These authors studied the hot electron generation efficiency as a function of the carrier lifetime and the particle size. A general feature of such a mechanism is that longer lifetimes result in the generation of hot carriers with large energies while larger diameters produce less energetic electrons at a fixed lifetime. Since the energy distribution of the hot electrons can dramatically change by simply varying any or both of these parameters, the diameter of the Au NP should have a significant influence on the internal quantum efficiency. Manjavacas et al. have theoretically found that for $10 \mathrm{~nm}$ diameter spherical silver nanoparticles plasmons decay into the most energetic hot carriers. Here, we conclude that in our experimental work, in which we investigated a different particle shape, material and environment compared to the work of Manjavacas et al., quantum effects do not play a significant role when decreasing the diameter of our asymmetrically shaped gold nanoparticle distributions from $30 \mathrm{~nm}$ to $10 \mathrm{~nm}$. The IQE reaches about $0.2 \%$, and the hot electron generation and injection efficiency are the same at longer wavelengths from $675 \mathrm{~nm}$ to $900 \mathrm{~nm}$ for our particle distributions centered around $10 \mathrm{~nm}$ and $30 \mathrm{~nm}$, respectively.

Interestingly, we observe a small photocurrent and an IQE of $0.02 \%$ in the $575 \mathrm{~nm}$ to $650 \mathrm{~nm}$ wavelength region for the $2 \mathrm{~nm}$ thick Au NPs but not for the $6 \mathrm{~nm}$ thick Au NPs. We attribute the photocurrent and the IQE in this wavelength region to the $2 \mathrm{~nm}$ to $5 \mathrm{~nm}$ diameter particles that are only present in our $2 \mathrm{~nm}$ thick Au NP samples, but not in the $6 \mathrm{~nm}$ thick Au NP samples. The measured IQE in this wavelength region is ten times lower than in the region above $650 \mathrm{~nm}$, and the particle size distribution from the SEM image suggests that about one third of the particles have diameters smaller than $5 \mathrm{~nm}$. Therefore, our data suggest that the IQE on a perparticle basis, i.e. the hot electron generation and injection efficiency per particle, is not enhanced for 2 to $5 \mathrm{~nm}$ diameter particles. For samples decorated with larger Au islands from 10 
$\mathrm{nm}$ thick evaporated $\mathrm{Au}$ films (Supporting Information S2), we observed only a small photocurrent less than $0.5 \mu \mathrm{A} / \mathrm{cm}^{2}$. These observations are consistent with predictions that larger size particles do generate less energetic hot carriers ${ }^{34}$.

We employed a heterogeneous particle size distribution on top of our spacer/reflector electrode to achieve broadband light absorption, in contrast to the simulated monodisperse $30 \mathrm{~nm}$ diameter Au particles which only support light absorption in a relatively narrow region with a FWHM of $100 \mathrm{~nm}$ centered around $830 \mathrm{~nm}$. The numerically calculated absorbed fraction for a periodic array of monodisperse $30 \mathrm{~nm}$ diameter gold nanoparticles can reach approximately $80 \%$ within the Au NPs and $95 \%$ in the total structure around $830 \mathrm{~nm}$ (FWHM of $100 \mathrm{~nm}$ ) (see Figure $4 \mathrm{a})$. The absorbed fraction could be further enhanced by e.g. coating the Au NPs by another dielectric material that better fulfills the critical coupling condition ${ }^{4}$. Experimentally we fabricated a heterogeneous particle size distribution with $\mathrm{Au}$ NP diameters of most frequent occurrence around $30 \mathrm{~nm}$ by annealing $6 \mathrm{~nm}$ thick $\mathrm{Au}$ films on top of $\mathrm{NiO}_{\mathrm{x}}$. Measurements from SEM images show that the distribution of particle diameters varies from $10 \mathrm{~nm}$ to $80 \mathrm{~nm}$. As expected from the broad particle size distribution, we observe a substantial spread in particle plasmon resonances leading to a substantial broadening of the experimentally measured absorbed fraction curve. In Figure $4 \mathrm{a}$ we show the measured total absorbed fraction in air of photocathodes with 6 nm thick Au NPs. These samples appear blue in color. As can be seen from the measured absorption data, the photoelectrode is an efficient light absorber at wavelengths above $\sim 500 \mathrm{~nm}$. We also show the simulated total absorbed fraction for a photocathode in water employing a periodic array of $30 \mathrm{~nm}, 20 \mathrm{~nm}, 10 \mathrm{~nm}$ diameter gold particles with a separation of $15 \mathrm{~nm}, 10$ $\mathrm{nm}, 5 \mathrm{~nm}$, respectively. Our simulations qualitatively support the notion that broadband absorption is possible in these structures. The simulations also show that there is no plasmon 
resonance peak below $600 \mathrm{~nm}$, in agreement with the experimentally observed low photocurrent in that wavelength range.

Figure $4 \mathrm{~b}$ shows the measured total absorbed fraction in air of photocathodes with $2 \mathrm{~nm}$ thick Au NPs. The samples with $2 \mathrm{~nm}$ thick Au NPs appear brown-reddish in color. The smaller absorbed fraction within the Au NPs is expected from our electromagnetic simulations and could be improved to similar values as for the $6 \mathrm{~nm}$ thick Au NPs by adjusting the $\mathrm{NiO}_{\mathrm{x}}$ spacer layer thickness to $80 \mathrm{~nm}$ (Supporting Information S9). However here we opted to keep the electrical properties of the two photoelectrode geometries the same and therefore did not alter the $\mathrm{NiO}_{\mathrm{x}}$ thickness. The SEM image of $2 \mathrm{~nm}$ thick Au NPs (Fig. 2b) yields a particle size distribution varying from $2 \mathrm{~nm}$ to $20 \mathrm{~nm}$. Generally, as the particles become more isotropic i.e. the aspect ratio of diameter to thickness tends towards unity, the plasmon resonance of the particles will blue-shift. Thus, for a random distribution of particles with diameters ranging from $2 \mathrm{~nm}$ to 20 $\mathrm{nm}$ and a thickness of $2 \mathrm{~nm}$, the plasmon peaks cover a region from around $600 \mathrm{~nm}$ to $900 \mathrm{~nm}$. The strongest plasmon peak is located around $800 \mathrm{~nm}$ since nanoparticles with $10 \mathrm{~nm}$ diameter have the highest occurrence. In addition smaller particles cause significantly smaller absorption because of their decreased volume.

Experimentally we observed a large broadband absorption feature from $400 \mathrm{~nm}$ to $550 \mathrm{~nm}$ for the $2 \mathrm{~nm}$ thick Au NPs sample which looks somewhat more pronounced than for the $6 \mathrm{~nm}$ thick Au NPs, and the $6 \mathrm{~nm}$ thick Au NPs sample shows unexpected low absorption around $400 \mathrm{~nm}$. Absorption in this region is due to the inter-band transitions in Au NPs and does not contribute to the generation of electrons that are energetic enough to drive the HER. Again our electromagnetic simulations qualitatively support broadband absorption, and show that no plasmon resonance peak exists below $600 \mathrm{~nm}$, not even for the smallest isotropic Au NPs in our 
$2 \mathrm{~nm}$ thick Au NP samples. We therefore speculate that the small photocurrents that are present in the region from $575 \mathrm{~nm}$ to $650 \mathrm{~nm}$ for the $2 \mathrm{~nm}$ thick Au NPs with diameters in the range from 2-5 nm, but not for the $6 \mathrm{~nm}$ thick larger diameter Au NPs, could be due to quantum confinement effects $^{42,43}$.

In summary, we present a new approach towards plasmon mediated hot-electron generation for solar-to-chemical energy conversion, in which hot-electrons are generated efficiently at the plasmon resonance frequency of gold nanoparticles. The cathodic photocurrents of our photoelectrode structures have a performance comparable to the best-published results ${ }^{8}$ albeit without the use of expensive platinum as a co-catalyst, much smaller catalytic surface areas and a minimum amount of gold. The key to achieve these large photocurrents is the design of an architecture that simultaneously enables improved light absorption and carrier separation, and utilizes small nanoparticles for efficient, energetic carrier generation. This work can provide the impetus for the design and fabrication of novel, yet simple, nanostructures for plasmon enhanced hot carrier chemistry. For the widespread deployment of hot carriers in catalysis, the quantum efficiency of hot carrier generation needs to be improved. A better understanding of the hot carrier spectra and lifetimes and a better mechanistic insight into catalytic processes will prove helpful in that respect.

'Supporting Information Available: Details of the fabrication process, the spectrum of the light source, thin film SEM characterization of the plasmonic photocathode and $\mathrm{NiO}_{\mathrm{x}}, \mathrm{XRD}$ characterization of the $\mathrm{NiO}_{\mathrm{x}}$ thin film, Electrochemical Impedance Spectroscopy (EIS) and optical characterization of $\mathrm{NiO}_{\mathrm{x}}$, electromagnetic simulations of photocathodes, photocurrent density response of plasmonic photocathodes and control experiments. This material is available free of charge via the Internet at http://pubs.acs.org/.' 
ACKNOWLEDGMENT We gratefully acknowledge support from the Robert A. Welch Foundation (Grant No. C-1825) and the National Science Foundation (NSF CAREER Award, CHE- 1352579). We gratefully acknowledge Luke H. Macfarlan and Sidan Fu for their contributions through the initial stages of this work.

\section{Author Contributions}

\# These authors contributed equally to this work.

\section{Notes}

The authors declare no competing financial interest.

\section{REFERENCES}

(1) Moskovits, M., Nat. Nanotechnol. 2015, 10 (1).

(2) Brongersma, M. L.; Halas, N. J.; Nordlander, P., Nat. Nanotechnol. 2015, 10 (1), 25-34.

(3) Linic, S.; Christopher, P.; Ingram, D. B., Nat. Mater. 2011, 10 (12), 911-921.

(4) Hägglund, C.; Zeltzer, G.; Ruiz, R.; Thomann, I.; Lee, H.-B.-R.; Brongersma, M. L.; Bent, S. F., Nano Lett. 2013, 13 (7), 3352-3357.

(5) Lee, J.; Mubeen, S.; Ji, X.; Stucky, G. D.; Moskovits, M., Nano Lett. 2012, 12 (9), 50145019 .

(6) Thomann, I.; Pinaud, B. A.; Chen, Z.; Clemens, B. M.; Jaramillo, T. F.; Brongersma, M. L., Nano Lett. 2011, 11 (8), 3440-3446.

(7) Warren, S. C.; Thimsen, E., Energy Environ. Sci. 2012, 5 (1), 5133-5146.

(8) Mubeen, S.; Lee, J.; Singh, N.; Kramer, S.; Stucky, G. D.; Moskovits, M., Nat. Nanotechnol. 2013, 8 (4), 247-251.

(9) Schweikhard, V.; Grubisic, A.; Baker, T. A.; Nesbitt, D. J., J. Phys. Chem. C 2011, 115 (1), 83-91.

(10) Schweikhard, V.; Grubisic, A.; Baker, T. A.; Thomann, I.; Nesbitt, D. J., Acs Nano 2011, 5 (5), 3724-3735.

(11) Liu, Z.; Hou, W.; Pavaskar, P.; Aykol, M.; Cronin, S. B., Nano Lett. 2011, 11 (3), 1111 1116.

(12) Li, J.; Cushing, S. K.; Zheng, P.; Meng, F.; Chu, D.; Wu, N., Nat. Commun. 2013, 4.

(13) Kim, H. J.; Lee, S. H.; Upadhye, A. A.; Ro, I.; Tejedor-Tejedor, M. I.; Anderson, M. A.; Kim, W. B.; Huber, G. W., ACS Nano 2014, 8 (10), 10756-10765. 
(14) Chen, H. M.; Chen, C. K.; Chen, C.-J.; Cheng, L.-C.; Wu, P. C.; Cheng, B. H.; Ho, Y. Z.; Tseng, M. L.; Hsu, Y.-Y.; Chan, T.-S.; Lee, J.-F.; Liu, R.-S.; Tsai, D. P., ACS Nano 2012, 6 (8), $7362-7372$.

(15) Hou, W. a. C., S. B., Adv. Funct. Mater. 2013, (23), 1612-1619.

(16) Sil, D.; Gilroy, K. D.; Niaux, A.; Boulesbaa, A.; Neretina, S.; Borguet, E., Acs Nano 2014, 8 (8), 7755-7762.

(17) Zhang, X. M.; Chen, Y. L.; Liu, R. S.; Tsai, D. P., Rep Prog Phys 2013, 76 (4).

(18) Li, J. T.; Cushing, S. K.; Zheng, P.; Meng, F. K.; Chu, D.; Wu, N. Q., Nat. Commun. 2013, 4 .

(19) Mukherjee, S.; Libisch, F.; Large, N.; Neumann, O.; Brown, L. V.; Cheng, J.; Lassiter, J. B.; Carter, E. A.; Nordlander, P.; Halas, N. J., Nano Lett. 2013, 13 (1), 240-247.

(20) Mukherjee, S.; Zhou, L.; Goodman, A. M.; Large, N.; Ayala-Orozco, C.; Zhang, Y.; Nordlander, P.; Halas, N. J., J. Am. Chem. Soc. 2014, 136 (1), 64-67.

(21) DuChene, J. S.; Sweeny, B. C.; Johnston-Peck, A. C.; Su, D.; Stach, E. A.; Wei, W. D., Angew Chem Int Edit 2014, 53, 7887-7891.

(22) Li, Z.; Ezhilarasu, G.; Chatzakis, I.; Dhall, R.; Chen, C.-C.; Cronin, S. B., Nano Lett. 2015, 15 (6), 3977-3982.

(23) Appavoo, K.; Wang, B.; Brady, N. F.; Seo, M.; Nag, J.; Prasankumar, R. P.; Hilton, D. J.; Pantelides, S. T.; Haglund, R. F., Nano Lett. 2014, 14 (3), 1127-1133.

(24) Sundararaman, R.; Narang, P.; Jermyn, A. S.; Goddard, W. A.; Atwater, H. A., Nat. Commun. 2014, 5.

(25) Park, J. Y.; Kim, S. M.; Lee, H.; Naik, B., Catal. Lett. 2014, 144 (12), 1996-2004.

(26) Clavero, C., Nat. Photonics 2014, 8 (2), 95-103.

(27) Knight, M. W.; Sobhani, H.; Nordlander, P.; Halas, N. J., Science 2011, 332 (6030), 702-

704.

(28) Fang, Z. Y.; Liu, Z.; Wang, Y. M.; Ajayan, P. M.; Nordlander, P.; Halas, N. J., Nano Lett. 2012, 12 (7), 3808-3813.

(29) Berini, P., Laser Photonics Rev. 2014, 8 (2), 197-220.

(30) Alavirad, M.; Mousavi, S. S.; Roy, L.; Berini, P., Opt Express 2013, 21 (4), 4328-4347.

(31) Kang, Y. M.; Gong, Y. J.; Hu, Z. J.; Li, Z. W.; Qiu, Z. W.; Zhu, X.; Ajayan, P. M.; Fang, Z. Y., Nanoscale 2015, 7 (10), 4482-4488.

(32) Baffou, G.; Quidant, R., Chem. Soc. Rev. 2014, 43 (11), 3898-3907.

(33) Wu, K. F.; Rodriguez-Cordoba, W. E.; Yang, Y.; Lian, T. Q., Nano Lett. 2013, 13 (11), 5255-5263.

(34) Manjavacas, A.; Liu, J. G.; Kulkarni, V.; Nordlander, P., ACS Nano 2014, 8 (8), 76307638.

(35) Hou, W.; Hung, W. H.; Pavaskar, P.; Goeppert, A.; Aykol, M.; Cronin, S. B., ACS Catal. 2011, 1 (8), 929-936.

(36) Qian, K.; Sweeny, B. C.; Johnston-Peck, A. C.; Niu, W. X.; Graham, J. O.; DuChene, J. S.; Qiu, J. J.; Wang, Y. C.; Engelhard, M. H.; Su, D.; Stach, E. A.; Wei, W. D., J. Am. Chem. Soc. 2014, 136 (28), 9842-9845.

(37) Chalabi, H.; Schoen, D.; Brongersma, M. L., Nano Lett. 2014, 14 (3), 1374-1380.

(38) Sun, K.; Park, N.; Sun, Z.; Zhou, J.; Wang, J.; Pang, X.; Shen, S.; Noh, S. Y.; Jing, Y.; Jin, S.; Yu, P. K. L.; Wang, D., Energy Environ. Sci. 2012, 5 (7), 7872-7877.

(39) Hagglund, C.; Apell, S. P., J. Phys. Chem. Lett. 2012, 3 (10), 1275-1285. 
(40) Lunkenheimer, P.; Loidl, A.; Ottermann, C. R.; Bange, K., Phys. Rev. B 1991, 44 (11), 5927-5930.

(41) Zhang, H.; Govorov, A. O., J. Phys. Chem. C 2014, 118 (14), 7606-7614.

(42) Scholl, J. A.; Koh, A. L.; Dionne, J. A., Nature 2012, 483 (7390), 421-U68.

(43) Ouyang, F.; Batson, P. E.; Isaacson, M., Phys. Rev. B 1992, 46 (23), 15421-15425. 


\section{Figure 1.}

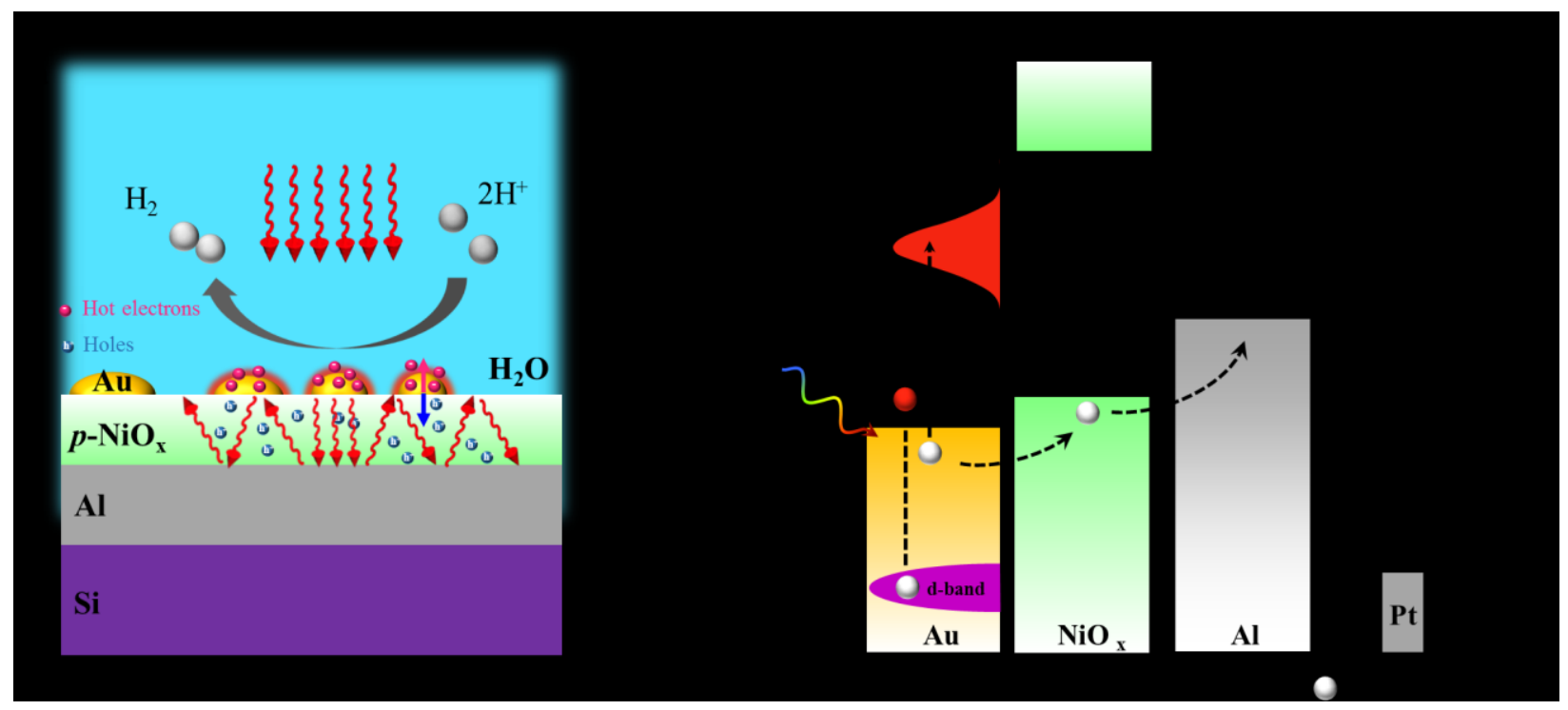

Figure 1. Structure and mechanism of operation of our plasmonic photocathode for plasmon mediated direct electron injection to drive solar-to-chemical energy conversion. a) Plasmonic photocathode for solar water splitting, consisting of plasmonic nanoparticles in direct contact with water, $\mathrm{p}-\mathrm{NiO}_{\mathrm{x}}$ as an electron blocking layer and aluminum as the electrode material. b) Energy schematic of our structure. Small metal nanoparticles facilitate the efficient decay of plasmons into hot carriers. Here the hot electron is directly injected into water molecules to drive the hydrogen evolution reaction. $\mathrm{A} \mathrm{NiO}_{\mathrm{x}}$ layer blocks electron transport while allowing hole transport to the platinum anode. Note that this novel plasmon-induced hot carrier generation mechanism for water splitting does not rely on a semiconductor/metal Schottky junction to collect hot-electrons. 


\section{Figure 2.}
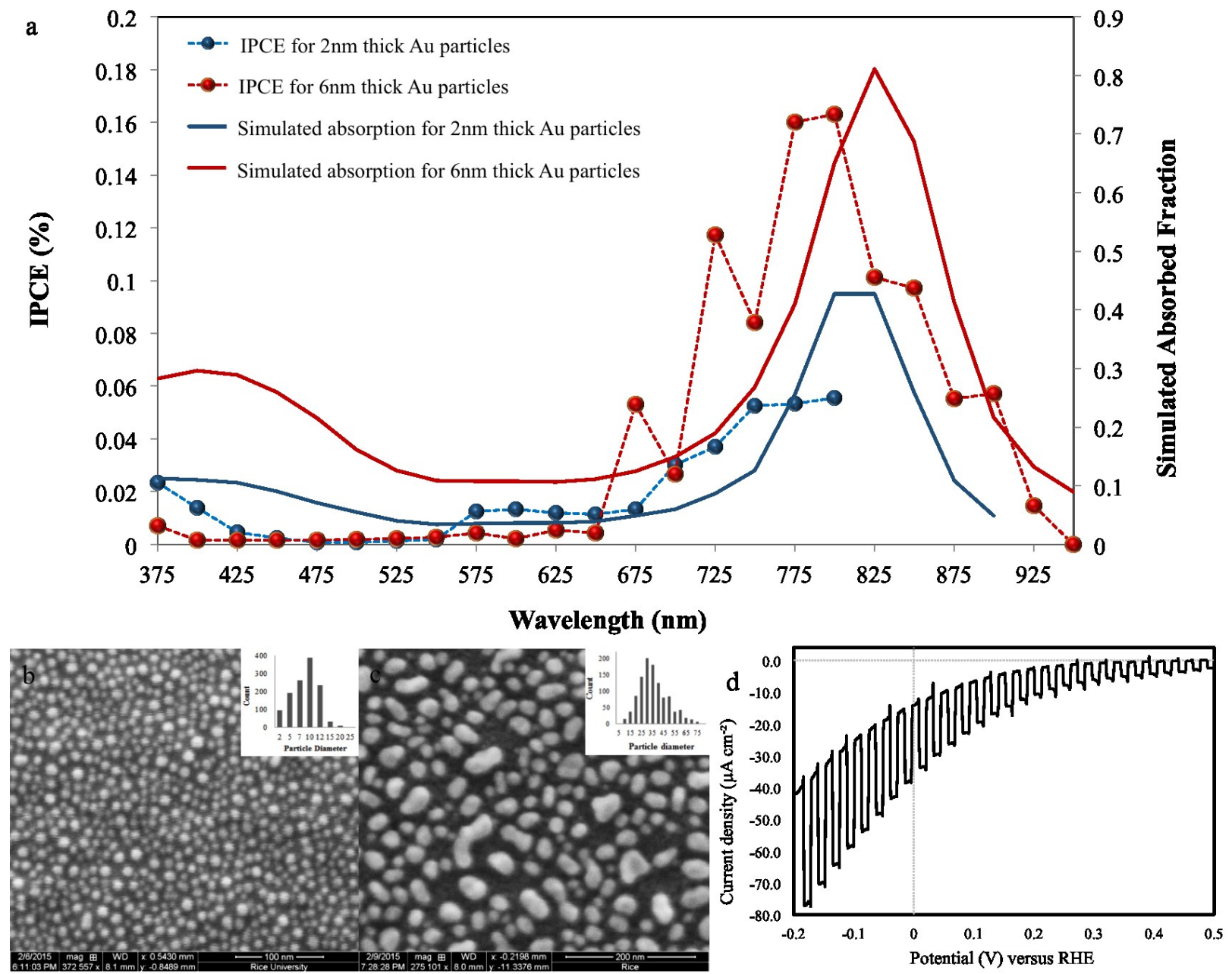

Figure 2. Photocatalytic performance of our structures. a) Comparison between measured IPCE (left axis) and simulated absorbed fraction (right axis) versus wavelength in $2 \mathrm{~nm}$ (blue) and $6 \mathrm{~nm}$ (red) thick gold nanoparticles. Note, electromagnetic simulations of the absorbed fraction were performed for gold nanoparticles with $10 \mathrm{~nm}(2 \mathrm{~nm}$ thick) and $30 \mathrm{~nm}$ diameter $(6 \mathrm{~nm}$ thick$)$, respectively. The spectral dependence of simulated and experimental data shows convincingly that the photocurrent originates from plasmon-induced hot carriers within the gold nanoparticles. b, c) SEM images and corresponding particle diameter distributions for particles formed from the deposition of a b) $2 \mathrm{~nm}$ and c) $6 \mathrm{~nm}$ thick Au film. 
d) Photocurrent generated from a plasmonic photocathode decorated with $2 \mathrm{~nm}$ thick particles in nitrogen-purged, $0.5 \mathrm{M} \mathrm{Na}_{2} \mathrm{SO}_{4}$ electrolyte buffered at $\mathrm{pH} 5.2$. The total white light illumination intensity was $100 \mathrm{~mW} \mathrm{~cm}{ }^{-2}$. The photocurrent was measured in a three-electrode photoelectrochemical cell configuration with a Pt mesh as the counter electrode, and is plotted versus the reference hydrogen electrode (RHE). The scan rate was $2 \mathrm{mV} / \mathrm{s}$. 


\section{Figure 3.}

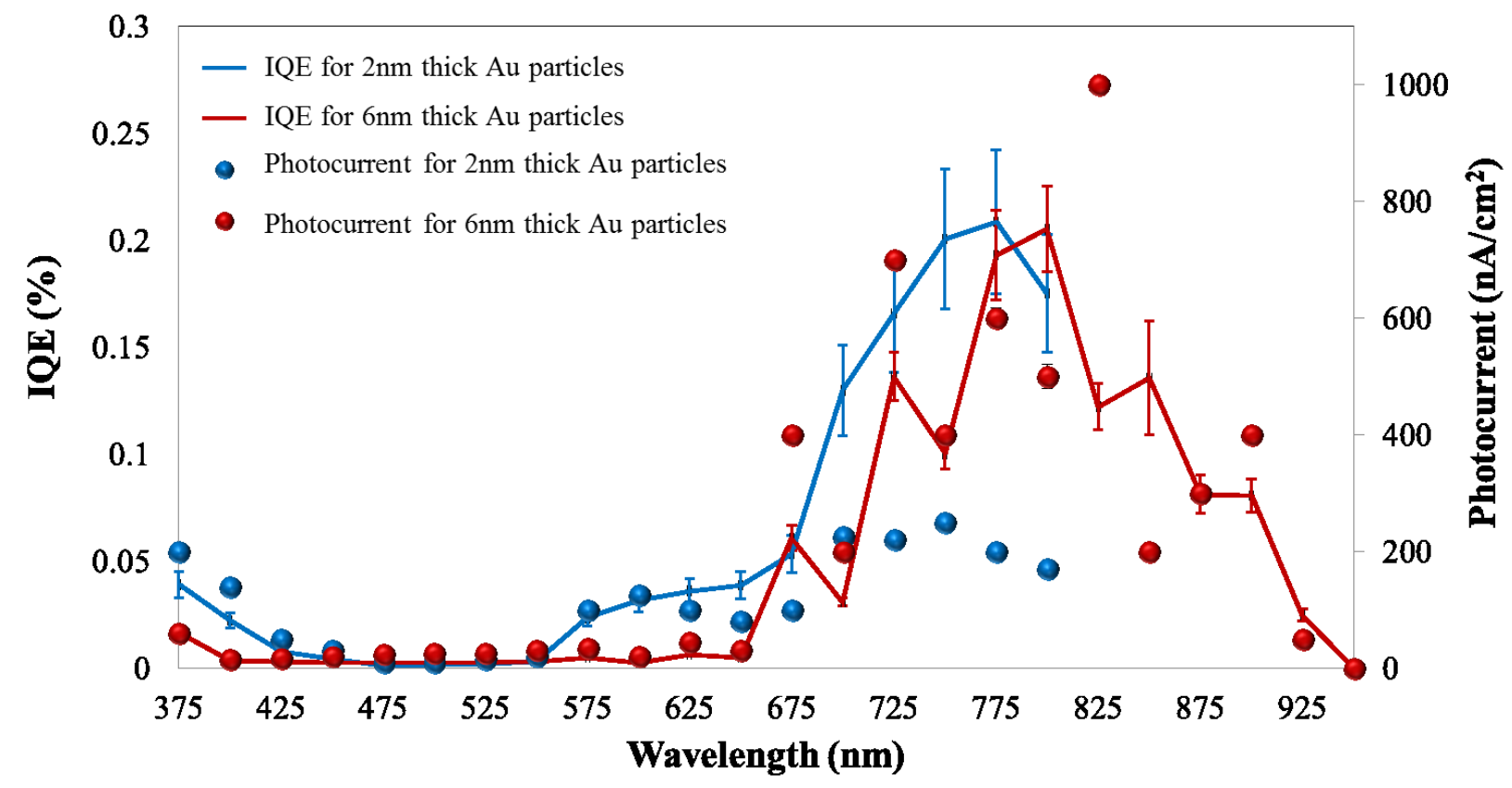

Figure 3. Comparison of the internal quantum efficiency (IQE) for small (10 nm diameter, $2 \mathrm{~nm}$ thick) and large (30 nm diameter, $6 \mathrm{~nm}$ thick) Au particles with the same plasmon resonance wavelength. The solid curve shows the internal quantum efficiency (left axis), and the symbols (right axis) show the measured photocurrent. We find that the IQE is comparable for small and large particles, suggesting that hot electron generation and injection efficiencies are similar and quantum size effects (i.e. wavefunction spill out) are not important for our $10 \mathrm{~nm}$ diameter gold particles. 


\section{Figure 4 .}

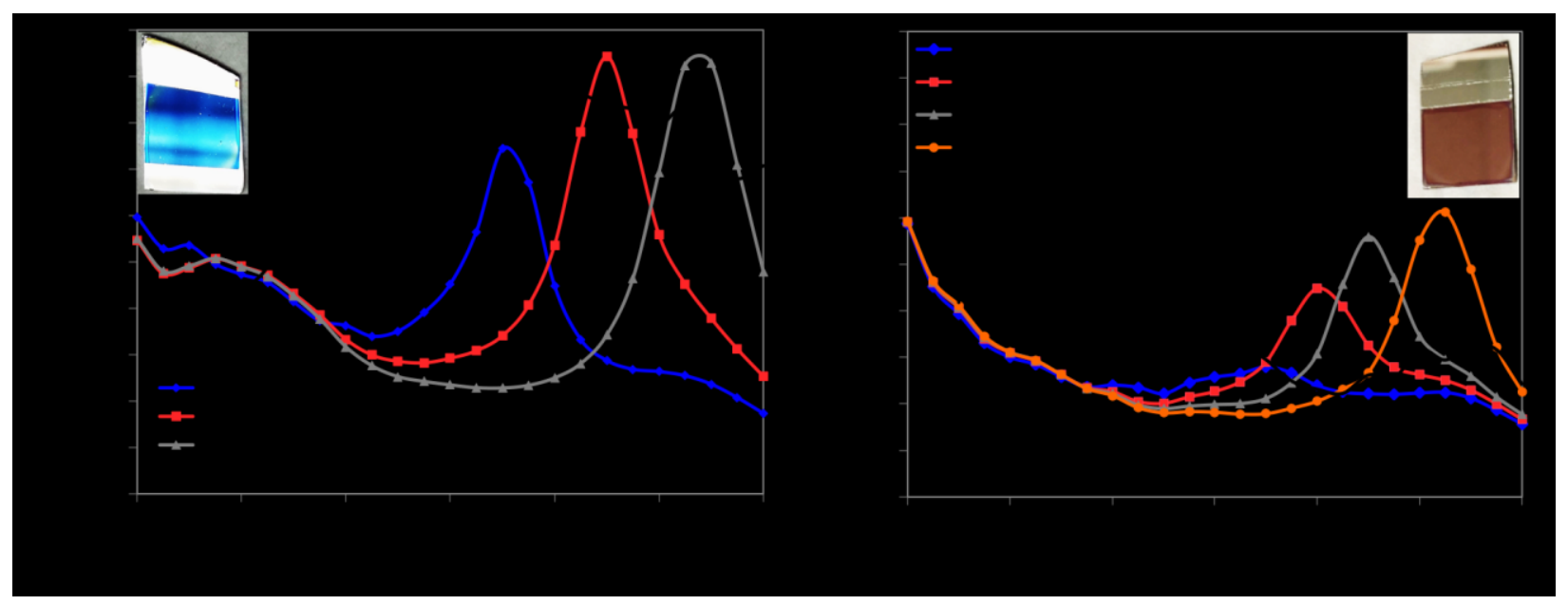

Figure 4. Experimentally measured total absorbed fraction for photocathode with $6 \mathrm{~nm}$ thick and $2 \mathrm{~nm}$ thick Au nanodisk in air versus simulated absorbed fraction of $6 \mathrm{~nm}$ thick and $2 \mathrm{~nm}$ thick Au nanodisks with different particle diameters.

a) Solid black line: experimentally measured total absorption for photocathode in air with $6 \mathrm{~nm}$ thick $\mathrm{Au}$ nanodisks. Colored lines with symbols: simulated total absorbed fraction for a photocathode in water employing a periodic array of $10 \mathrm{~nm}, 20 \mathrm{~nm}$, and $30 \mathrm{~nm}$ diameter gold particles with a separation of $5 \mathrm{~nm}, 10 \mathrm{~nm}$, and $15 \mathrm{~nm}$ respectively. b) Solid black line: experimentally measured total absorption for photocathode in air with $2 \mathrm{~nm}$ thick Au nanodisks. Colored lines with symbols: simulated total absorbed fraction for a photocathode in water employing a periodic array of $2 \mathrm{~nm}, 5 \mathrm{~nm}, 7 \mathrm{~nm}$, and $10 \mathrm{~nm}$ diameter gold particles with a separation of $1 \mathrm{~nm}, 2.5 \mathrm{~nm}, 3.5 \mathrm{~nm}$, and $5 \mathrm{~nm}$ respectively. The diameters of particles were chosen based on the calculated particle size distribution from SEM characterization. 
For TOC only

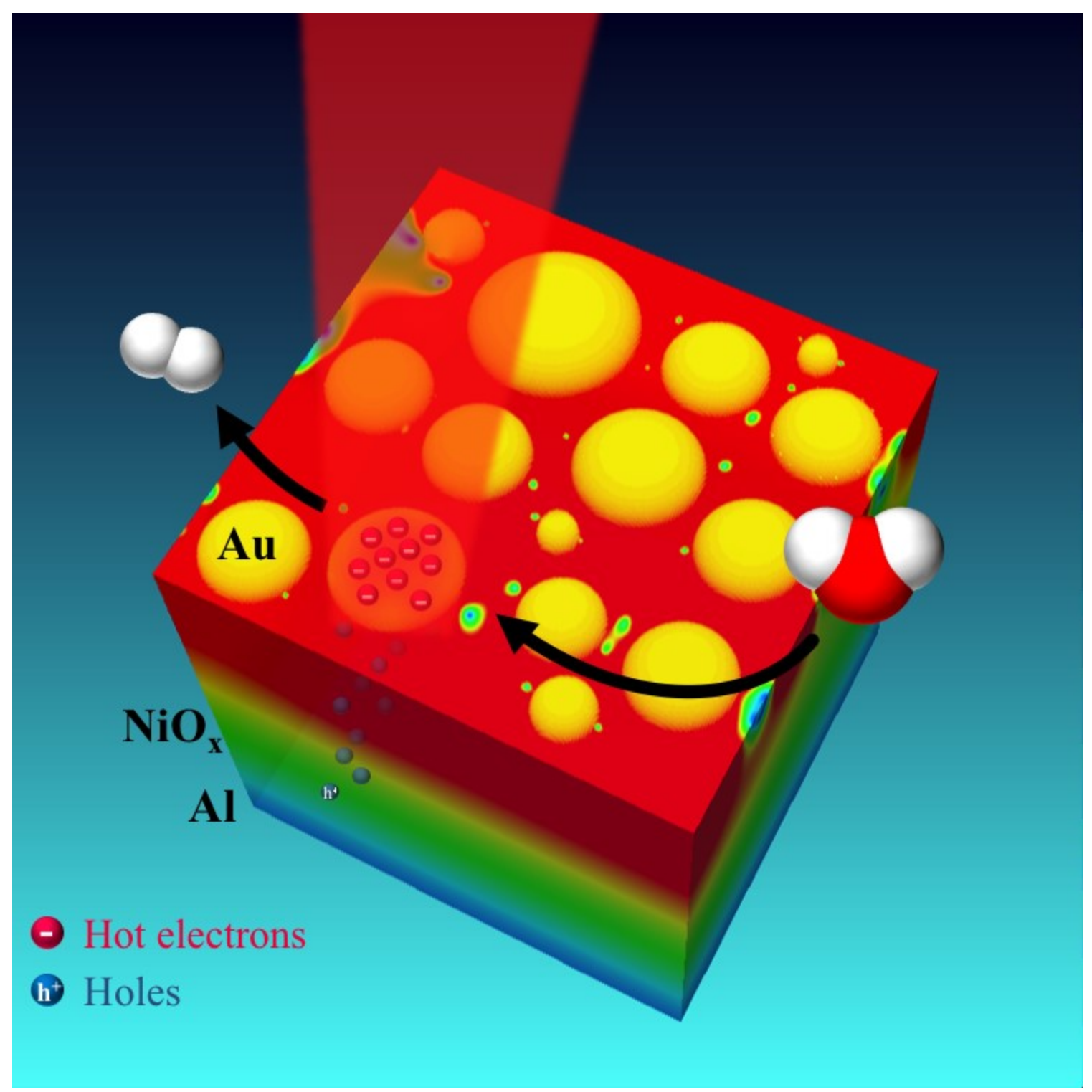

